\title{
ESCALA DIAGRAMÁTICA PARA AVALIAÇÃO DA SEVERIDADE DA MANCHA ALVO EM FOLHAS DE PEPINEIRO ${ }^{1}$
}

\author{
Adriana Teramoto ${ }^{2}$, Renata Alves Aguiar ${ }^{2}$, \\ Riccely Ávila Garcia², Marise Cagnin Martins ${ }^{3}$, Marcos Gomes Cunha ${ }^{2}$
}

\begin{abstract}
DIAGRAMMATIC SCALE TO EVALUATE

TARGET SPOT SEVERITY IN CUCUMBER PLANT LEAVES

The lack of a diagrammatic scale to evaluate the cucumber target spot, caused by Corynespora cassiicola, led to the development of an assessment system for a faster and more reliable way to quantify this disease. So, a diagrammatic scale was elaborated considering the maximum and minimum severity limits of the disease observed in the field. The intermediate values followed logarithmic increments for the target spot symptoms $(0.3 \%, 0.8 \%, 2 \%, 5 \%, 11.5 \%, 25 \%$, and $46 \%)$. For validating the scale, eight raters quantified the disease severity on fifty leaves infected with different levels of the disease. Initially, the severity estimation was carried out without the aid of the scale. Then, the same raters used it to estimate the severity on the same leaves previously assessed. Assessments with the aid of the diagrammatic scale were more precise and accurate, according to most of the raters. The proposed diagrammatic scale was considered adequate to estimate the target spot severity in cucumber leaves, and it can be used in epidemiologic studies and in evaluation strategies for controlling this disease.
\end{abstract}

KEY-WORDS: Corynespora cassiicola (Berk. \& Curt.) C.T. Wei; Cucumis sativus L.; pathometry; plant disease epidemiology.

\section{INTRODUÇÃO}

O fungo Corynespora cassiicola (Berk. \& Curt.) C.T. Wei infecta folhas, flores, frutos, raízes e ramos, e já foi relatado em mais de 312 hospedeiros, em regiões tropicais e subtropicais (Farr et al. 2009). No Brasil, a mancha alvo vem ocorrendo, com certa frequência, nos Estados de São Paulo e Paraná, em pepinos do tipo 'japonês', cultivados em estufas (Martins et al. 2003, Verzignassi et al. 2003), e no Estado de Goiás, também em pepino japonês, em campo aberto (Teramoto et al. 2006).

\section{RESUMO}

Devido à ausência de escala diagramática para a mancha alvo do pepineiro, causada por Corynespora cassiicola, verificouse a necessidade de um sistema de quantificação da doença, que proporcionasse maior rapidez e precisão na obtenção dos resultados. Para tanto, uma escala diagramática foi elaborada, considerando-se o limite máximo e mínimo de severidade da doença observados no campo. Os valores intermediários da escala seguiram incrementos logarítmicos para os sintomas da mancha alvo $(0,3 \% ; 0,8 \% ; 2 \% ; 5 \% ; 11,5 \% ; 25 \%$; e $46 \%)$. Para a validação da escala, oito avaliadores quantificaram a severidade da doença, em cinquenta folhas com diferentes níveis de doença. Inicialmente, a estimativa da severidade foi feita sem auxílio da escala. A seguir, os mesmos avaliadores, utilizando a escala diagramática proposta, estimaram a severidade nas mesmas folhas avaliadas anteriormente. As avaliações com a escala diagramática foram mais precisas e acuradas, nas estimativas da maioria dos avaliadores. A escala diagramática proposta foi considerada adequada para estimar a severidade da mancha alvo, em folhas de pepineiro, e poderá ser utilizada em estudos epidemiológicos e de avaliação de estratégias de controle desta doença.

PALAVRAS-CHAVE: Corynespora cassiicola (Berk. \& Curt.) C.T. Wei; Cucumis sativus L.; patometria; epidemiologia.

Os primeiros sintomas da mancha alvo, em folhas de pepineiro, são pequenas manchas de coloração clara, que evoluem para manchas angulares, com o centro de cor palha, circundadas por pequeno halo amarelo claro. Posteriormente, as manchas crescem, tomando formato arredondado e apresentando centro marrom claro e bordos encharcados de coloração olivácea. O coalescimento das manchas pode provocar seca do limbo foliar, com consequente desfolha da planta (Martins et al. 2003, Verzignassi et al. 2003), podendo acarretar perdas de até $60 \%$ na produção.

1. Trabalho recebido em out./2010 e aceito para publicação em set./2011 (nº registro: PAT 12017/ DOI: 10.5216/pat.v41i3.12017).

2. Universidade Federal de Goiás, Escola de Agronomia e Engenharia de Alimentos, Goiânia, GO, Brasil.

E-mails: adritera@terra.com.br, renatalvesufg@yahoo.com.br, riccelyavila@yahoo.com.br,mgc@agro.ufg.br.

3. Instituto Biológico, Centro Experimental Central, Campinas, SP, Brasil. E-mail: marise@biologico.sp.gov.br. 
Um fato preocupante, no manejo da mancha alvo do pepineiro, é que os fungicidas utilizados para o controle de outras manchas foliares, em cucurbitáceas, têm sido ineficientes para seu controle. Além disto, as práticas culturais correntes não reduzem a severidade da doença e os híbridos utilizados atualmente são bastante susceptíveis (Verzignassi et al. 2003). Neste contexto, o uso de escala diagramática, principal ferramenta na avaliação da severidade de manchas foliares, é muito importante para quantificação de doenças e necessário tanto para o estudo de medidas de controle, na determinação da eficiência de um fungicida ou na caracterização da resistência varietal, quanto para a epidemiologia, na construção de curvas de progresso da doença e estimativa dos danos provocados por ela (Amorim 1995).

Já existem escalas diagramáticas para avaliação da severidade de doenças para diversas hortaliças, como tomateiro (Boff et al. 1991, Mello et al. 1997), alface (O' Brien \& Van Bruggen 1992), inhame (Michereff et al. 2000), melancia (Halfeld-Vieira \& Nechet 2006), mandioquinha-salsa (Mesquini et al. 2009) e, inclusive, para o pepineiro (Azevedo 1997), porém, nenhum trabalho contemplou a mancha alvo em pepineiro. Por esta razão, o objetivo deste trabalho foi elaborar uma escala diagramática de fácil utilização e que reproduza resultados precisos e acurados, visando a auxiliar estudos epidemiológicos e de controle de Corynespora cassiicola em pepineiro, em virtude do aumento da importância desta doença e da falta de informações sobre a interação pepineiro-Corynespora-ambiente.

\section{MATERIAL E MÉTODOS}

\section{Elaboração da escala diagramática}

Foram coletadas, aleatoriamente, 80 folhas de pepineiro do híbrido 'Yoshinari', com diferentes níveis de severidade da mancha alvo, em campo de produção localizado no município de Bela Vista (GO), em março de 2009. Este híbrido foi escolhido pelo produtor por possuir boa produtividade e frutos com boa aceitação comercial, porém, assim como outros híbridos comerciais, também é susceptível à C. cassiicola.

No laboratório do Núcleo de Pesquisa em Fitopatologia da Escola de Agronomia e Engenharia de Alimentos da Universidade Federal de Goiás (EA/ UFG), as folhas foram escaneadas, em equipamento
HP scanjet 2400. Com o auxílio do Programa Quant (Vale et al. 2003), as folhas escaneadas passaram por um processo em que as lesões foram circundadas e coloridas, de forma a se obter um contraste com a parte sadia, e, a partir daí, pôde-se quantificar a severidade real, em cada folha. Assim, foi determinado o valor máximo e mínimo da severidade da doença, com os valores intermediários calculados segundo incrementos logarítmicos, obedecendo à lei de acuidade visual de Weber-Fechner (Horsfall \& Barratt 1945).

\section{Validação da escala diagramática}

Para os testes de validação da escala diagramática, foram selecionadas cinquenta imagens de folhas digitalizadas, abrangendo diferentes graus de severidade. Oito avaliadores sem experiência quantificaram a severidade da doença, a partir das imagens, as quais foram projetadas aleatoriamente, e estimaram a severidade da mancha alvo do pepineiro, sem o auxílio da escala proposta. A seguir, os mesmos avaliadores, utilizando a escala diagramática proposta, estimaram a severidade nas mesmas imagens de folhas avaliadas anteriormente.

A acurácia e a precisão de cada avaliador foram determinadas por meio de equações de regressão linear simples, com a severidade real obtida como variável independente e a severidade estimada como variável dependente. Posteriormente, a acurácia das estimativas de cada avaliador foi determinada pelo teste $t$, aplicado ao intercepto da regressão linear $(a)$ e ao coeficiente angular da reta $(b)$, para testar as hipóteses $\mathrm{H}_{0}: a=0 \mathrm{e}$ $\mathrm{H}_{1}: b=1$, a $5 \%$. Valores de intercepto significativamente diferentes de zero descrevem a presença de desvios constantes, enquanto valores de coeficientes angulares da reta significativamente diferentes de um indicam a presença de desvios sistemáticos. A precisão dos avaliadores foi obtida pelos coeficientes de determinação da regressão $\left(R^{2}\right)$ e pela distribuição dos resíduos. A reprodutibilidade das estimativas foi determinada por valores de $R^{2}$ obtidos de regressões lineares, entre as severidades estimadas pelos diferentes avaliadores, combinados aos pares (Campbell \& Madden 1990, Nutter Junior et al. 1993, Nutter Junior \& Schultz 1995). As análises de regressão foram realizadas com o auxílio do software estatístico SAS ${ }^{\circledR}$.

\section{RESULTADOS E DISCUSSÃO}

A escala diagramática proposta apresenta sete níveis de severidade: $0,3 \% ; 0,8 \% ; 2 \% ; 5 \% ; 11,5 \%$; 
$25 \%$; e $46 \%$, para os sintomas de mancha alvo em pepineiro (Figura 1). A escala foi confeccionada a partir de imagens digitalizadas de folhas com os respectivos níveis de severidade, como realizado por Díaz et al. (2001), Halfeld-Vieira \& Nechet (2006), Salgado et al. (2009) e Soares et al. (2009).

A severidade mínima e máxima encontradas para a mancha alvo foi de $0,3 \%$ e $46,0 \%$, respectivamente. $\mathrm{O}$ valor máximo de severidade da doença não está de acordo com as observações de Kranz (1977), em que muitas mensurações em campo envolvem níveis de severidade inferiores a $30 \%$. Porém, em várias outras escalas diagramáticas, é possível encontrar valores máximos superiores a 30\% (Amorim et al. 1993, Godoy et al. 1997, Mello et al. 1997, Díaz et al. 2001, Leite \& Amorim 2002, Martins et al. 2004, Angelotti et al. 2008, Mesquini et al. 2009, Salgado et al. 2009, Soares et al. 2009). Estes valores apenas refletem a severidade máxima da doença encontrada no campo, o que pode variar, dependendo da relação patógeno-hospedeiro, sem, contudo, interferir na acurácia e precisão da escala diagramática confeccionada a partir destes valores.
A proximidade entre a estimativa e os valores reais determina a acurácia das avaliações, que é definida como a exatidão de uma medida isenta de erros sistemáticos e que pode ser medida pelo coeficiente angular $(b)$ e o intercepto da regressão linear $(a)$, entre a severidade real e a estimada (Bergamin Filho \& Amorim 1996). De acordo com Nutter Junior et al. (1993), o coeficiente angular (b) deve ser igual a um (1), sem desvios sistemáticos, e o coeficiente linear (a) deve ser igual a zero (0). Sendo assim, os avaliadores que utilizaram a escala podem ser considerados acurados, pois os valores de $a$ não foram significativamente diferentes de zero (0), com exceção do Avaliador $\mathrm{B}$, e os de $b$ não foram significativamente diferentes de um (1), excetuando-se os Avaliadores A, C e D (Tabela 1).

A precisão, que é definida como a exatidão de uma operação, em que há rigor ou refinamento na medida (Bergamin Filho \& Amorim 1996), é um fator relevante a ser considerado na validação de uma escala diagramática, além da acurácia. A precisão pode ser avaliada por meio do coeficiente de determinação da regressão e pela variação dos erros absolutos (Nutter Junior \& Schultz 1995). Todos os

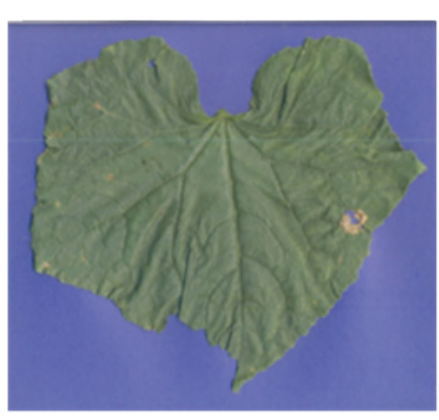

$0,3 \%$

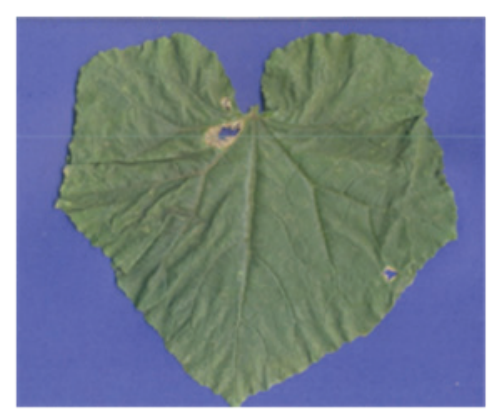

$0,8 \%$

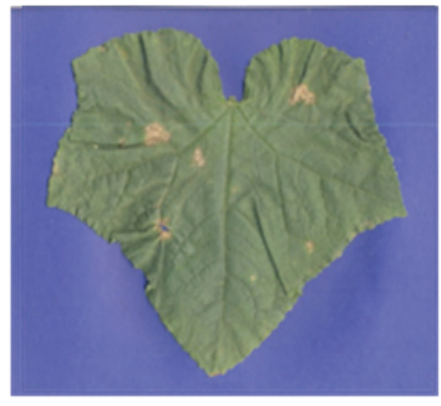

$2,0 \%$

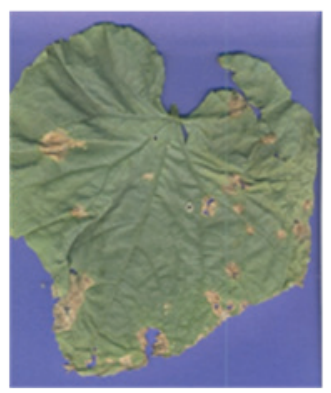

$5,0 \%$

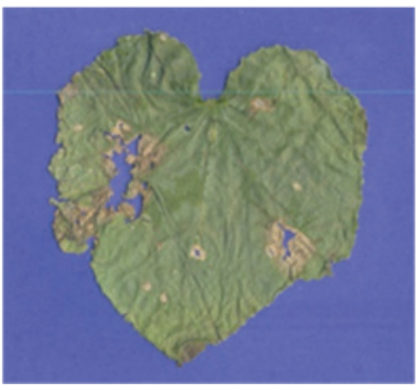

$11,5 \%$

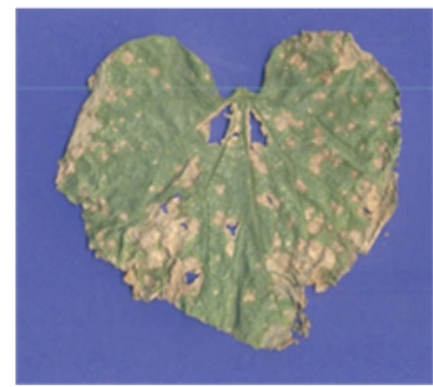

$25,0 \%$

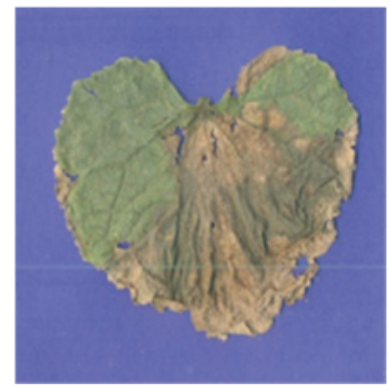

$46,0 \%$

Figura 1. Escala diagramática de severidade (\% da área foliar afetada), para mancha alvo do pepineiro (Cucumis sativus) causada por Corynespora cassiicola (Goiânia, GO, 2009). 
Tabela 1. Coeficientes lineares $(a)$, angulares $(b)$ e de determinação $\left(R^{2}\right)$ das regressões entre severidade real (variável dependente) e severidade estimada (variável dependente), para cada avaliador, antes e depois da utilização da escala diagramática para avaliação da severidade da mancha alvo, em folhas de pepineiro (Goiânia, GO, 2009).

\begin{tabular}{ccccccc}
\hline \multirow{2}{*}{ Avaliador } & \multicolumn{3}{c}{ Sem escala } & \multicolumn{3}{c}{ Com escala } \\
\cline { 2 - 6 } & $a$ & $b$ & $R^{2}$ & $a$ & \multicolumn{3}{c}{$B$} & $R^{2}$ \\
\hline $\mathrm{A}$ & $4,95^{*}$ & 1,67 & 0,52 & 2,06 & $0,92^{*}$ & 0,82 \\
$\mathrm{~B}$ & 17,09 & $1,21^{*}$ & 0,38 & $0,51^{*}$ & 0,90 & 0,90 \\
$\mathrm{C}$ & 3,08 & 0,93 & 0,72 & 1,58 & $0,99^{*}$ & 0,91 \\
$\mathrm{D}$ & 2,72 & $1,04^{*}$ & 0,78 & 1,80 & $0,97^{*}$ & 0,89 \\
$\mathrm{E}$ & 25,81 & $0,81^{*}$ & 0,14 & 1,47 & 0,85 & 0,84 \\
$\mathrm{~F}$ & 18,20 & 1,27 & 0,15 & 2,23 & 0,72 & 0,73 \\
$\mathrm{G}$ & 49,01 & $1,14^{*}$ & 0,19 & 2,74 & 1,04 & 0,78 \\
$\mathrm{H}$ & 6,31 & $1,21^{*}$ & 0,61 & 1,74 & 0,89 & 0,86 \\
\hline Média & & 0,44 & & 0,84 \\
\hline
\end{tabular}

* A hipótese da nulidade $(a=0$ ou $b=1)$ foi rejeitada pelo teste $t(\mathrm{p}=0,05)$.

avaliadores apresentaram boa precisão em suas avaliações, utilizando a escala proposta, variando entre 0,73 e 0,91 , sendo a média de 0,84 . Valor similar ou inferior a este também foi encontrado por outros autores (Michereff et al. 1998, Michereff et al. 2000, Díaz et al. 2001, Martins et al. 2004, Andrade et al. 2005, Belasque Júnior et al. 2005), apesar de o valor desejável, segundo Kranz (1988), ser 0,95.

Os avaliadores B, E, F e $\mathrm{H}$ subestimaram a severidade da doença, avaliada com o uso da escala diagramática proposta, e apenas o avaliador G superestimou-a. A tendência de os avaliadores subestimarem também foi verificada em estudos semelhantes com outros patossistemas (Michereff et al. 1998, Michereff et al. 2000, Halfeld-Vieira \& Nechet 2006, Sussel et al. 2009). A solução para se corrigir este fato seria treinar os avaliadores (Nutter Junior \& Schultz 1995, Godoy et al. 1997).

Com relação aos erros absolutos (diferença entre a severidade estimada e a real, pelos diversos avaliadores), houve redução dos valores das estimativas com o auxílio da escala diagramática, quando comparados com os valores dos erros absolutos das estimativas obtidas sem a utilização da escala. Com a utilização da escala diagramática, os erros absolutos não apresentaram padrão tendencioso, refletindo na superestimação ou subestimação da severidade, e os mesmos variaram na avaliação sem utilização da escala e com a escala entre -33,4 e 84,4, e -21,4 e 17,8, respectivamente (Figuras 2).

A grande maioria dos erros absolutos dos avaliadores que utilizaram a escala foi inferior a $5 \%$. Os avaliadores obtiveram maiores erros na faixa de $20-40 \%$ de severidade, muito provavelmente pelo fato de o padrão de lesão dos $46 \%$ ser contínuo, ou seja, ter havido o coalescimento das lesões menores. Mesmo assim, segundo o critério utilizado nos programas Distrain (Tormelin \& Howell 1988) e Dispro (Nutter Junior \& Worawitlikit 1989), um avaliador é considerado excelente quando o erro da estimativa está compreendido dentro de um intervalo de 5\% acima ou abaixo do real e bom quando ele não ultrapassa o valor de $10 \%$. Com base neste critério, a maioria das estimativas dos oito avaliadores pode ser considerada boa.

Além da acurácia e da precisão, outro indicador da eficiência da escala diagramática desenvolvida é a reprodutibilidade das avaliações entre os avaliadores (Berger 1980). Diferentes avaliadores devem estimar os mesmos valores de severidade, utilizando a mesma escala, para avaliação do mesmo material (Nutter Junior \& Schultz 1995). Sem a utilização da escala diagramática, o coeficiente de determinação $\left(R^{2}\right)$ das regressões das estimativas entre os pares de avaliadores variou de 0,14 a 0,83 , com média de 0,47 (Tabela 2). Com a utilização da escala diagramática, os valores de $R^{2}$ variaram de 0,64 a 0,91 , com média de 0,80 , assemelhando-se ao constatado na validação de escalas diagramáticas para outros patossistemas (Martins et al. 2004, Díaz et al. 2001). Sendo assim, a reprodutibilidade das avaliações foi garantida com o uso da escala diagramática.

A padronização da avaliação da severidade da mancha alvo do pepineiro é desejável e necessária, pois a uniformização da metodologia de avaliação permitirá comparar resultados obtidos em experimentos em diferentes locais, variedades e métodos de controle, dentre outros. 

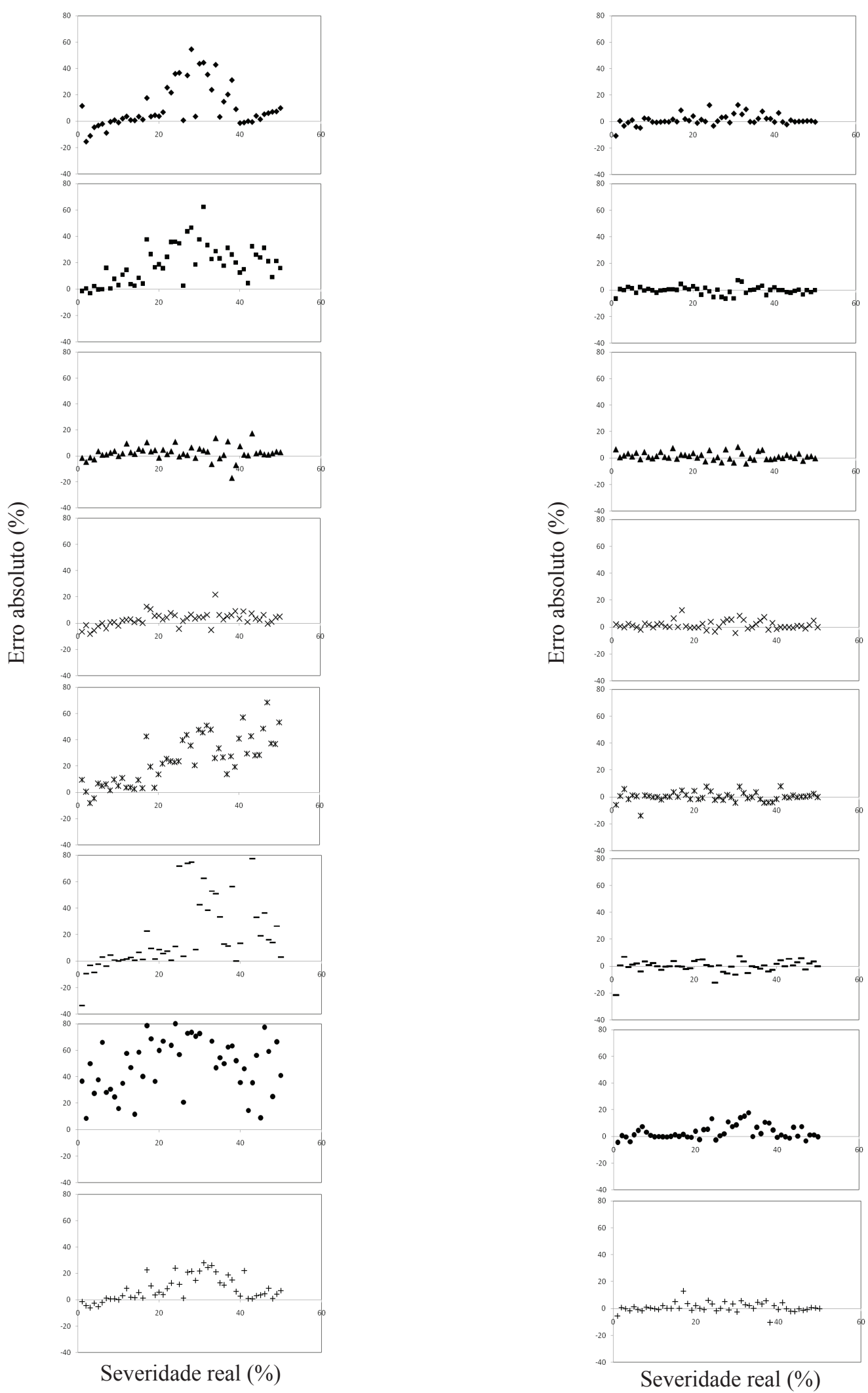

Figura 2. Erros absolutos (severidade estimada menos a severidade real) para oito avaliadores da mancha alvo (Corynespora cassiicola) de pepineiro (Cucumis sativus). Gráficos à esquerda representam os erros absolutos sem o uso da escala diagramática e os da direita com o uso da escala. Avaliadores: $\bullet=\mathrm{A}, \boldsymbol{\nabla}=\mathrm{B}, \boldsymbol{\Delta}=\mathrm{C}, \mathrm{X}=\mathrm{D}, *=\mathrm{E},-=\mathrm{F}, \bullet=\mathrm{G}$ e $+=\mathrm{H}$ (Goiânia, GO, 2009). 
Tabela 2. Coeficiente de determinação $\left(R^{2}\right)$ de equações de regressão linear relacionando as estimativas da mancha alvo causada por Corynespora cassiicola, em folhas de pepineiro, entre avaliadores sem e com auxílio da escala diagramática (Goiânia, GO, 2009).

\begin{tabular}{|c|c|c|c|c|c|c|c|}
\hline \multicolumn{8}{|c|}{ Sem escala } \\
\hline Avaliador & $\mathrm{B}$ & $\mathrm{C}$ & $\mathrm{D}$ & $\mathrm{E}$ & $\mathrm{F}$ & $\mathrm{G}$ & $\mathrm{H}$ \\
\hline $\mathrm{A}$ & 0,78 & 0,48 & 0,60 & 0,37 & 0,39 & 0,53 & 0,83 \\
\hline B & & 0,41 & 0,54 & 0,46 & 0,46 & 0,57 & 0,77 \\
\hline $\mathrm{C}$ & & & 0,73 & 0,14 & 0,14 & 0,18 & 0,53 \\
\hline $\mathrm{D}$ & & & & 0,26 & 0,24 & 0,30 & 0,71 \\
\hline $\mathrm{E}$ & & & & & 0,47 & 0,25 & 0,43 \\
\hline $\mathrm{F}$ & & & & & & 0,17 & 0,40 \\
\hline G & & & & & & & 0,53 \\
\hline $\mathrm{H}$ & & & & & & & \\
\hline \multicolumn{8}{|c|}{ Com escala } \\
\hline Avaliador & $\mathrm{B}$ & $\mathrm{C}$ & $\mathrm{D}$ & $E$ & $\mathrm{~F}$ & $\mathrm{G}$ & $\mathrm{H}$ \\
\hline $\mathrm{A}$ & 0,81 & 0,78 & 0,84 & 0,81 & 0,70 & 0,87 & 0,85 \\
\hline B & & 0,87 & 0,88 & 0,82 & 0,80 & 0,75 & 0,88 \\
\hline $\mathrm{C}$ & & & 0,92 & 0,82 & 0,71 & 0,74 & 0,82 \\
\hline $\mathrm{D}$ & & & & 0,82 & 0,70 & 0,76 & 0,91 \\
\hline E & & & & & 0,76 & 0,70 & 0,85 \\
\hline $\mathrm{F}$ & & & & & & 0,64 & 0,69 \\
\hline G & & & & & & & 0,73 \\
\hline $\mathrm{H}$ & & & & & & & \\
\hline
\end{tabular}

\section{CONCLUSÃO}

A escala diagramática proposta para avaliação da severidade da mancha alvo em pepineiro, causada por Corynespora cassiicola, foi de fácil utilização, proporcionando boa acurácia, precisão e reprodutibilidade das estimativas, constituindo-se, assim, em mais uma ferramenta a ser utilizada em estudos epidemiológicos e de avaliação de estratégias de controle desta doença.

\section{AGRADECIMENTOS}

Aos graduandos do Curso de Agronomia da EA/UFG, pelo auxílio na validação da escala diagramática, e ao Dr. Ângelo A. B. Sussel, pesquisador da Embrapa Cerrados, pelo auxílio estatístico.

\section{REFERÊNCIAS}

AMORIM, L. Avaliação de doenças. In: BERGAMIN FILHO, A.; KIMATI, H.; AMORIM, L. (Eds.). Manual de fitopatologia: princípios e conceitos. 3. ed. São Paulo: Agronômica Ceres, 1995. p. 647-671.
AMORIM, L. et al. Clorose variegada dos citros: uma escala diagramática para avaliação da severidade da doença. Fitopatologia Brasileira, Brasília, DF, v. 18, n. 2, p. 174-180, 1993.

ANDRADE, G. C. G. et al. Escala diagramática para avaliação da severidade da mancha foliar do eucalipto causada por Quambalaria eucalypti. Fitopatologia Brasileira, Brasília, DF, v. 30, n. 5, p. 504-509, 2005.

ANGELOTTI, F. et al. Diagrammatic scale for assessment of grapevine rust. Tropical Plant Pathology, Brasília, DF, v. 33, n. 6, p. 439-443, 2008.

AZEVEDO, L. A. S. Manual de quantificação de doenças de plantas. São Paulo: LASA, 1997.

BELASQUE JÚNIOR, J. et al. Escalas diagramáticas para avaliação da severidade do cancro cítrico. Fitopatologia Brasileira, Brasília, DF, v. 30, n. 4, p. 387-393, 2005.

BERGAMIN FILHO, A.; AMORIM, L. Doenças de plantas tropicais: epidemiologia e controle econômico. Piracicaba: Agronômica Ceres, 1996.

BERGER, R. D. Measuring disease intensity. In: TENG, P. S.; KRUPA, S. V. (Eds.). Crop loss assessment. St. Paul: University of Minnesota, 1980.

BOFF, P.; ZAMBOLIM, L.; VALE, F. X. R. Escalas para avaliação de severidade de mancha de estenfilio (Stemphylium solani) e da pinta preta (Alternaria solani) em tomateiro. Fitopatologia Brasileira, Brasília, DF, v. 16, n. 3, p. 280-283, 1991.

CAMPBELL, C. L.; MADDEN, L. V. Introduction to plant disease epidemiology. New York: Wiley Interscience, 1990.

DÍAZ, C. G.; BASSANEZI, R. B.; BERGAMIN FILHO, A. Desenvolvimento e validação de uma escala diagramática para Xanthomonas axonopidis pv. phaseoli em feijoeiro. Summa Phytopathologica, Botucatu, v. 27, n.1, p. 35-39, 2001.

FARR, D. F. et al. Fungal databases. 2009. Disponível em: $<$ http://nt.ars-grin.gov/fungaldatabases $>$. Acesso em: 8 fev. 2010.

GODOY, C. V. et al. Diagrammatic scales for bean diseases: development and validation. Zeitschrift für Pflanzenkrankheinten und Pflanzenschutz, Stuttgart, v. 104, n. 4, p. 336-345, 1997.

HALFELD-VIEIRA, B. A.; NECHET, K. L. Elaboração e validação de escala diagramática para avaliação da mancha-de-cercospora em melancia. Fitopatologia Brasileira, Brasília, DF, v. 31, n. 1, p. 46-50, 2006.

HORSFALL, J. G.; BARRAT, R. W. An improved grading system for measuring plant disease. Phytopathology, St. Paul, n. 35, p. 655, 1945. 
KRANZ, J. A study in maximum severity in plant diseases. In: TRAVEUX DÉDIÉS À GEORGES VIENNOTBOURGIN, 1977, Paris. Proceedings... Paris: Socièté Française de Phytopathologie, 1977. p. 167-173.

KRANZ, J. Measuring plant disease. In: KRANZ, J.; ROTEM, J. (Eds.). Experimental techniques in plant disease epidemiology. Heidelberg: Springer-Verlag, 1988.

LEITE, R. M. V. B. C.; AMORIM, L. Elaboração e validação de escala diagramática para mancha de Alternaria em girassol. Summa Phytopathologica, Botucatu, v. 28, n. 1, p. 14-19, 2002.

MARTINS, M. C. et al. Escala diagramática para a quantificação do complexo de doenças foliares de final de ciclo em soja. Fitopatologia Brasileira, Brasília, DF, v. 29, n. 2, p. 179-184, 2004.

MARTINS, M. C. et al. Ocorrência da mancha-alvo causada por Corynespora cassicola em pepino (Cucumis sativus) no Brasil. Fitopatologia Brasileira, Brasília, DF, v. 28, n. 1, p. 208, 2003.

MELLO, S. C. M.; TAKATSU, A.; LOPES, C. A. Escala diagramática para avaliação da mancha-bacteriana do tomateiro. Fitopatologia Brasileira, Brasília, DF, v. 22, n. 4, p. 447-448, 1997.

MESQUINI, R. M. et al. Escala diagramática para a quantificação de Septoria apiicola e Cercospora arracina em mandioquinha-salsa. Tropical Plant Pathology, Brasília, DF, v. 34, n. 4, p. 250-255, 2009.

MICHEREFF, S. J.; MAFFIA, L. A.; NORONHA, M. A. Escala diagramática para avaliação da severidade da queima das folhas do inhame. Fitopatologia Brasileira, Brasília, DF, v. 25, n. 4, p. 612-619, 2000.

MICHEREFF, S. J. et al. Escala diagramática e tamanho de amostras para avaliação da severidade da mancha parda da mandioca (Cercosporidium henningsii). Agrotrópica, Ilhéus, v. 10, n. 3, p. 143-148, 1998.

NUTTER JUNIOR, F. W. et al. Assessing the accuracy, intra-rater repeatability, and inter-rater reliability of disease assessment systems. Phytopathology, St. Paul, v. 83, n. 8, p. 806-812, 1993.

NUTTER JUNIOR, F. W.; SCHULTZ, P. M. Improving the accuracy and precision of disease assessments: selection of methods and use of computer-aided training programs. Canadian Journal of Plant Pathology, Burnaby, v. 17, n. 1, p. 174-184, 1995.
NUTTER JUNIOR, F. W.; WORAWITLIKIT, O. Disease.Pro: a computer program for evaluating and improving a person ability to assess disease proportion. Phytopathology, St. Paul, v. 79, n. 10, p. 1135, 1989.

O'BRIEN, R. D.; VAN BRUGGEN, A. H. C. Accuracy, precision, and correlation to yield of disease severity scales for corky root of lettuce. Phytopathology, St. Paul, v. 82, n. 1, p. 91-96, 1992.

SALGADO, M. et al. Escala diagramática para avaliação da severidade da mancha de Phoma do cafeeiro. Tropical Plant Pathology, Brasília, DF, v. 34, n. 6, p. 422-427, 2009.

SOARES, R. M.; GODOY, C. V.; OLIVEIRA, M. C. N. Escala diagramática para avaliação da severidade da mancha alvo da soja. Tropical Plant Pathology, Brasília, DF, v. 34, n. 5, p. 333-338, 2009.

SUSSEL, A. A. B.; POZZA E. A.; CASTRO, H. A. Elaboração e validação de escala diagramática para avaliação da severidade do mofo cinzento em mamoneira. Tropical Plant Pathology, Brasília, DF, v. 34, n. 3, p. 186191, 2009.

TERAMOTO, A.; CAVALCANTE, P. R.; CUNHA, M. G. Primeiro relato da ocorrência de Corynespora cassiicola na cultura do pepino em Goiás. Fitopatologia Brasileira, Brasília, DF, v. 31, n. 1, p. 151, 2006.

TOMERLIN, J. R.; HOWELL, T. A. Distrain: a computer program for training people to estimate disease severity on cereal leaves. Plant Disease, St. Paul, v. 72, n. 5, p. 455459, 1988.

VALE, F. X. R. et al. A software plant disease severity assessment. In: INTERNATIONAL CONGRESS OF PLANT PATHOLOGY, 8., 2003, Christchurch. Proceedings... Christchurch: Plant Pathology Society, 2003. p. 105.

VERZIGNASSI, J. R.; VIDA, J. B.; TESSMANN, D. J. Corynespora cassicola causando epidemias de manchas foliares em pepino 'japonês' sob estufa no norte do Paraná. Fitopatologia Brasileira, Brasília, DF, v. 28, n. 5, p. 570, 2003. 